\title{
Neutrino Masses or New Interactions
}

\section{Bruce H. J. McKellar* and M. Garbutt ${ }^{\dagger}$}

University of Melbourne, Parkville, Victoria 3052, Australia

E-mail: 'b.mckeliar@physics unimelb.edu. aúl and mag@physics.unimeib.edu. aù

\section{G. J. Stephenson Jr. $\ddagger$}

University of New Mexico, Albuquerque, New Mexico 87131

E-mailigj j@baryon.phys.unm.edui

\section{T. Goldman ${ }^{\S}$}

Los Alamos National Laboratory, Los Alamos, New Mexico 87545

E-mail'goldmanets.lanl.govi

ABstract: Recent proposals to study the mass of the "electron" neutrino at a sensititvity of $0.3 \mathrm{eV}$ can be used to place limits on the right handed and scalar charged currents at a level which improves on the present experimental limits. Omission of such interactions from the analysis can lead to the inference of an incorrect value for the mass.

\section{Introduction}

Understanding the properties of the neutrino is one of the foremost problems in modern particle physics. Indications from neutrino oscillation experiments are that at least one neutrino has a non-zero mass, but only differences in the squared masses can be determined in this way. The atmospheric neutrino data measured at the Super-Kamiokande experiment favors $\nu_{\mu}-\nu_{\tau}$ oscillation with a mass difference of $\delta m_{\text {atm }}^{2} \sim 3 \times 10^{-3} \mathrm{eV}^{2}$ [i] interpretation of this is that one neutrino has a mass $m_{3} \geq 0.05 \mathrm{eV}$, the first hint of physics beyond the Standard Model (SM).

The absolute neutrino mass scale is accessible through the kinematics of weak interactions. A program of Tritium beta decay spectral measurements has been pursued to this end for a number of years [2] $]$. Originally the focus of this program was to determine the Lorentz structure of Fermi's theory of weak interactions [3i]. More recently the focus

\footnotetext{
${ }^{*}$ Speaker.

${ }^{\dagger}$ supported in part by the Australian Research Council

${ }^{\ddagger}$ supported in part by the National Science Foundation

${ }^{\S}$ supported in part by the Department of Energy under contract W-7405-ENG-36
} 
has been solely on determining the mass of the electron anti-neutrino through precision measurements of the end-point of the electron energy spectrum [4] The current upper bound on the neutrino mass set by these experiments is $m_{\nu_{e}} \leq 2.5 \mathrm{eV}$ while future experiments plan to achieve a sub-eV sensitivity of $0.3 \mathrm{eV}$ [יi].

Many extensions to the minimal Standard Model introduce interactions with Lorentz structures other than V-A which have a coupling on a weaker scale than the Fermi coupling. In this note we examine the consequences of non-standard currents for the interpretation of the measured value of the electron neutrino mass, extending the work of Ref. [i $[\overline{6}]$, to which we refer for details of the notation used here. In particular we show that, in the presence of neutrino mixing, the non-standard currents may produce interference effects which completely distort conclusions drawn from the experiments concerning the nature of physics beyond the Standard Model.

\section{Formalism}

The new, non-standard interactions will in general have different Lorentz transformation properties from the dominant $V-A$ structure. For convenience we recast the usual $S, P, T, V$ and $A$ currents into their right and left handed components $S_{R}, S_{L}, T, R$ and $L$ with $L=(V-A), R=(V+A), S_{R}=(S+P)$ and $S_{L}=(S-P)$, described by the elements

$$
\Gamma_{S_{L}}=\left(1-\gamma_{5}\right), \quad \Gamma_{S_{R}}=\left(1+\gamma_{5}\right), \quad \Gamma_{R}=\gamma^{\mu} \Gamma_{S_{R}}, \quad \Gamma_{L}=\gamma^{\mu} \Gamma_{S_{L}}, \quad \Gamma_{T}=\left[\gamma^{\mu}, \gamma^{\nu}\right] / 2
$$

in the Dirac Algebra. In the case of negatively charged lepton decay the subscripts of the operators $S_{R}$ and $S_{L}$ denote the chirality of the neutrino field and do not correspond to the chirality of the negatively charged lepton field.

The most general effective interaction Hamiltonian for low energy, semi-leptonic decays is written as

$$
H_{I}=\sum_{\alpha, \beta=S_{L}, S_{R}, R, L, T} G^{\alpha \beta} \sum_{f}\left(J_{h \alpha}^{\dagger} . J_{f \beta}+\text { h.c. }\right)
$$

where $f=e, \mu, \tau$, labels the weak eigenstate and in the Tritium case $J_{f \lambda=e \lambda}=\bar{\psi}_{e} \Gamma_{\lambda} \psi_{\nu_{e}}$ represents the leptonic current while $J_{h \alpha}$ represents the hadronic current. In the SM the only couplings present are $\beta=L$ and $\alpha=L, R$.

To allow for neutrino mixing, we allow the weak eigenstates $\nu_{f}$ to be linear combinations of the mass eigenstates,

$$
\nu^{f}=\sum_{i} \cos \theta_{k}^{f} \nu_{k}
$$

where the $\cos \theta_{k}^{f}$ are the direction cosines in the coordinate system spanned by the mass eigenstates ${ }^{1}$.

\footnotetext{
${ }^{1}$ For simplicity we do not consider $C P$ violation in the neutrino mixing matrix.
} 
In general, should additional currents exist, the boson mediating the current need not couple the eigenstates of the new interaction to the same linear combination of mass eigenstates; hence we define

$$
\hat{\nu}_{X}^{f}=\sum_{i} \cos \theta_{k X}^{f} \nu_{k}
$$

where the subscript ' $\mathrm{X}$ ' corresponds to the type of interaction and takes the values $S_{R}, S_{L}, R$, or $T$.

The new interactions produce interference terms in the beta decay spectra proportional

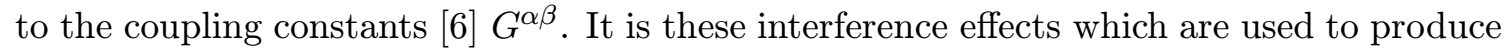
the best limits on the strength of the new couplings [i];]

The strength of interference effects are usually evaluated under the assumption that the weak eigenstate is the same as the mass eigenstate, and quoted relative to the strength of the SM coupling as,

$$
\rho_{X}=\frac{\hat{g}^{2} M_{W}^{2}}{g^{2} M_{X}^{2}}
$$

where $\hat{g}$ and $M_{X}$ are the coupling constant and mass of the non-SM boson being exchanged Recent limits are given as $[\bar{i}]$

$$
\rho_{R} \leq 0.07, \quad \rho_{S_{R}} \leq 0.1, \quad \rho_{S_{L}} \leq 0.01
$$

The constraints on the left-chiral scalar interaction are stronger than the others as this current would produce a charged lepton of the wrong chirality. Because of this strong constraint we do not consider this interaction further.

If the assumption that the weak and mass eigenstates are the same is relaxed, the effective strength of the interaction is modified by the mixing matrices of the standard and the non-standard currents.

\section{Spectra}

In this section we show how new Lorentz structures will be detected in the various experimental spectra. The differential electron energy spectrum with scalar and right-handed currents involves a sum over atomic/molecular final states, $i$, which can be simplified near the end point where the product $F\left(E_{\beta}\right) q_{\beta}$, and the ratio $m_{e} / E_{\beta}$ are nearly constant.

The effects of a finite neutrino mass on the differential spectrum is to cause a distortion near the end point, as the spectrum falls off as $E_{\nu} q_{\nu}$ rather than $E_{\nu}^{2}$, and a shift of the end point by $m_{\nu}\left(\mathcal{E}_{0} \rightarrow \mathcal{E}_{0}-m_{\nu}\right)$. Neutrino mixing has a similar result, producing several kinks in the spectrum at the point where the electron energy is such that decays to the $k^{\text {th }}$ mass

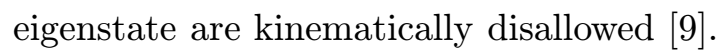

Recent and proposed experiments measure an integral spectrum over all beta energies above some value $E_{\beta}^{C}, \sum_{i} N_{i}\left(E_{\beta}^{C}\right)$. In the Mainz experiment results for a number of different values of $E_{\beta}^{C}$ are combined in a weighted average; the data is then fitted as a function of $E_{l}<E_{\beta}^{C}$, a lowest cutoff energy. The weighting takes into account the systematics of the 
spectrometer [i⿺廴in] insight from a crude model of this process, in which we integrate the integral spectrum from $E_{l}$ to infinity,

$$
G_{i}\left(E_{l}\right)=\int_{E_{l}}^{\infty} N_{i}\left(E_{\beta}^{C}\right) d E_{\beta}^{C} \approx \frac{K^{\prime}}{12} \sum_{k}\left(1+\epsilon_{k}\right) E_{\nu_{l} i}^{4}\left[1-3 \frac{m_{k}^{2}}{E_{\nu_{l} i}^{2}}+2 \frac{\phi_{k}}{E_{\nu_{l} i}}\right],
$$

introducing $E_{\nu_{l} i}=\mathcal{E}_{0}^{i}-E_{l}$, the neutrino energy in the $i^{\text {th }}$ channel, and making the approximation $E_{\nu_{l} i} \gg m_{k}$, which is accurate to about one part in a thousand for cutoff energies $E_{\nu_{l} i} / m_{k}>35$, and deteriorates rapidly for energies $E_{\nu_{l} i} / m_{k}<10$. Our results are expressed in terms of

$$
\begin{aligned}
\epsilon_{k X} & =\rho_{X} \frac{\cos \theta_{k X}}{\cos \theta_{k}}, \quad \text { and } \epsilon_{\mathrm{k}}=\epsilon_{\mathrm{kR}}^{2}+\epsilon_{\mathrm{kS}}^{2}\left(\frac{\mathrm{G}_{\mathrm{V}}^{2}}{\mathrm{G}_{\mathrm{V}}^{2}+3 \mathrm{G}_{\mathrm{A}}^{2}}\right) \text { and } \\
\phi_{k} & =-2 \frac{m_{k}}{\left(1+\epsilon_{k}\right)}\left[\frac{m_{e}}{<E_{\beta}>} \epsilon_{k R}+\epsilon_{k S_{R}}\left(\frac{G_{V}^{2}}{G_{V}^{2}+3 G_{A}^{2}}\right)\right] .
\end{aligned}
$$

To simplify the discussion we examine the case of a decay to one final atomic/molecular state, $i=0$. Further we restrict the discussion to only one mass eigenstate $k=1$. With these simplifications the function to be fitted to the data will be

$$
G_{0}\left(E_{l}\right) \approx \frac{\mathcal{A}}{12}\left[E_{\nu_{l} i}^{4}-3 E_{\nu_{l} i}^{2} m_{1}^{2}+2 E_{\nu_{l} i}^{3} \phi_{1}+\mathcal{B} E_{\nu_{l} i}\right]
$$

The overall magnitude $\mathcal{A}=\left(1+\epsilon_{1}\right) \cos ^{2} \theta_{1}$ will be determined from the data, as will the end point $\mathcal{E}_{0}^{0}$. The background $\mathcal{B}$ can be independently determined from measurements far from the end point.

In fitting the data it is important to be sure that all of the parameters, including $\mathcal{A}, \mathcal{B}$ and $\mathcal{E}_{0}^{0}$ are physically reasonable, and then to find a minimum $\chi^{2}$ at a point on the $\left(m_{1}^{2}, \phi_{1}\right)$ plane consistent with the independent limits on $\epsilon_{k}$. Discussions of the experimental results of future experiments should include a discussion of the values of all of the fitted parameters.

We now generalize to the case where all mass eigenstates participate in the decay. The KATRIN experiment should be sensitive to a mass of $0.3 \mathrm{eV}$, or about a $0.02 \%$ shift in $G_{0}\left(E_{l}\right)$ at an energy of $E_{\nu_{l} i} \approx 40 \mathrm{eV}$. We investigate the influence of new interactions by taking values of $\cos \theta_{k}$ and also the mass differences between the mass eigenstates consistent with the LMA solution to the solar neutrino problem. The absolute value of the relative difference between the spectra with $\left(G_{0}^{R}\right)$ and without $\left(G_{0}^{\prime}\right)$ right-handed vector couplings, $\Delta G=\left(G_{0}^{R}-G_{0}^{\prime}\right) / G_{0}^{\prime}$ over the space of the non-standard direction cosines have been plotted in the $\left(\cos \theta_{2 R}, \cos \theta_{3 R}\right)$ plane.

On the $\Delta G=0$ curve in the plane there is no difference between the standard and nonstandard spectra as a result of destructive interference between different mass eigenstates. Even the non-observation of interference effects in spectra or correlation parameters could be misleading. The magnitude of the relative difference between spectra for some values $\cos \theta_{k X}$ is larger than the $0.02 \%$ detection threshold. Ignorance of mixing and non-standard interactions in this situation would result in an incorrect fit for the value of $m_{\nu_{e}}$. The possibility of weaker interactions in beta-decay must be taken into account when analysing future precision results. We must be ready to be surprised by the data! 


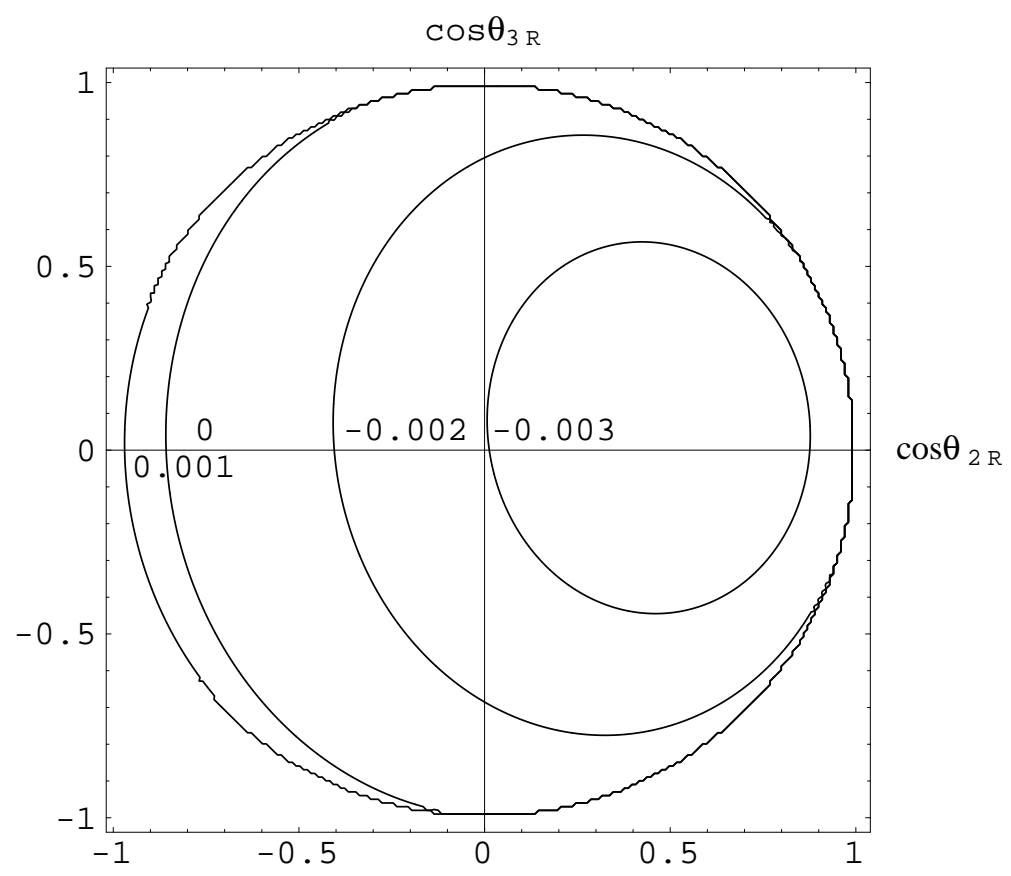

Figure 1: Constant value contours of $\Delta G$ for the right-handed vector interaction. In this plot the third mass eigenstate has a mass of $m_{3}=1 \mathrm{eV}$. The numbers immediately to the right of each contour give the value of $\Delta G$. The 0.001 contour reaches furthest to the left.

\section{References}

[1] Y. Fukuda et al. [Super-Kamiokande Collaboration], Phys. Rev. Lett. 81 (1998) 1562; [hep-ex/9807003].

[2] M. Fritschi et al., Phys. Lett. B 173 (1986) 485. R. G. H. Robertson, et al., Phys. Rev. Lett. 67 (1991) 957. A. I. Belesev et al., Phys. Lett. B 350 (1995) 263. V. M. Lobashev et al., Phys. Lett. B 460 (1999) 227. C. Weinheimer et al., Phys. Lett. B 300 (1993) 210.

[3] O. Kofoed-Hansen, Kgl. Danske Videnskab. Selskab, Mat.-fys. Medd. 28, No. 9 (1954).

[4] C. Weinheimer et al., Phys. Lett. B 460 (1999) 219.

[5] V. M. Lobashev et al., Nucl. Phys. Proc. Suppl. 91 (2001) 280.

[6] G. J. Stephenson, T. Goldman and B. H. J. McKellar, Phys. Rev. D 62 (2000) 093013; [hep-ph/0006095]. G. J. Stephenson and T. Goldman, Phys. Lett. B 440 (1998) 89; [nucl-th/9807057].

[7] E. G. Adelberger et al. [ISOLDE Collaboration], Phys. Rev. Lett. 83 (1999) 1299 [Erratum-ibid. 83 (1999) 3101]

[8] P. Herczeg, In Langacker, P. (ed.): Precision tests of the standard electroweak model, pp $786-83 \%$.

[9] B. H. J. McKellar, Phys. Lett. B 97 (1980) 93; R. E. Shrock, Phys. Lett. B 96 (1980) 159. 